\title{
Quality Metrics of a Fecal Immunochemical Test-Based Colorectal Cancer Screening Program in Korea
}

Dae Ho Kim¹, Jae Myung Cha², Min Seob Kwak², Jin Young Yoon ${ }^{1,2}$, Young-Hak Cho², Jung Won Jeon², Hyun Phil Shin², Kwang Ro Joo ${ }^{2}$, and Joung II Lee ${ }^{2}$

${ }^{\prime}$ Department of Medicine, Graduate School, Kyung Hee University, and ${ }^{2}$ Department of Internal Medicine, Kyung Hee University Hospital at Gangdong, Kyung Hee University School of Medicine, Seoul, Korea

Background/Aims: Knowledge regarding the quality metrics of fecal immunochemical test (FIT)-based colorectal cancer screening programs is limited. The aim of this study was to investigate the performance and quality metrics of a FITbased screening program. Methods: In our screening program, asymptomatic subjects aged $\geq 50$ years underwent an annual FIT, and subjects with positive FIT results underwent a subsequent colonoscopy. The performance of the FIT and colonoscopy was analyzed in individuals with a positive FIT who completed the program between 2009 and 2015 at a university hospital. Results: Among the 51,439 screened participants, $75.1 \%$ completed the FIT. The positive rate was $1.1 \%$, and the colonoscopy completion rate in these patients was $68.6 \%$. The positive predictive values of cancer and advanced neoplasia were $5.5 \%$ and $19.1 \%$, respectively. The adenoma detection rate in the patients who underwent colonoscopy after a positive FIT was $48.2 \%$ (60.0\% for men and $33.6 \%$ for women). The group with the highest tertile quantitative FIT level showed a significantly higher detection rate of advanced neoplasia than the group with the lowest tertile (odds ratio, 2.6; 95\% confidence interval, 1.4 to $5.1 ; p<0.001)$. Conclusions: The quality metrics used in the United States and Europe may be directly introduced to other countries, including Korea. However, the optimal quality metrics should be established in each country. (Gut Liver 2018;12:183-189)

Key Words: Colonoscopy; Colorectal neoplasms; Fecal occult blood test; Quality; Screening

\section{INTRODUCTION}

The incidence of colorectal cancer (CRC) has increased rapidly in many Asian countries, including Korea. ${ }^{1}$ The National Cancer Screening Program (NCSP) of Korea provides a single annual fecal immunochemical test (FIT) as a primary screening tool for adults aged $\geq 50$ years, and a confirmatory colonoscopy as a second step for those with a positive FIT result., ${ }^{2,3}$ The efficacy of a FIT-based population screening program may ultimately depend on the completion rate of FIT and colonoscopy. Furthermore, the adoption of quality improvement measures and continuous quality assessment is essential to improve the current effectiveness of this screening program. ${ }^{4}$ However, little is known about the quality indicators of the FIT-based CRC screening program. Recently, quality metrics for FIT were recommended in the United States, ${ }^{5}$ however, few studies that used these recommended measures are available. In addition, quality indicators for colonoscopy were revised in the United States and Europe, ${ }^{4,6,7}$ but updated quality indicators were focused on primary screening colonoscopy and not on secondary colonoscopy after FIT positive results. Therefore, measurement of quality indicators for a FIT-based CRC screening program may help us identify areas for quality improvement of this program.

The aim of this study was to investigate the performance and quality metrics of the FIT-based CRC screening program. To the best of our knowledge, this is the first study to assess this issue.

\section{MATERIALS AND METHODS}

\section{Patients}

In this study, asymptomatic subjects aged $\geq 50$ years who performed an annual FIT in the CRC screening program in a uni-

\footnotetext{
Correspondence to: Jae Myung Cha

Department of Internal Medicine, Kyung Hee University Hospital at Gangdong, Kyung Hee University School of Medicine, 892 Dongnam-ro, Gangdong-gu, Seoul 05278, Korea

Tel: +82-2-440-6113, Fax: +82-2-440-6295, E-mail: drcha@khu.ac.kr

Received on January 15, 2017. Revised on May 1, 2017. Accepted on June 19, 2017. Published online December 8, 2017

pISSN 1976-2283 eISSN 2005-1212 https://doi.org/10.5009/gnl17030

(a) This is an Open Access article distributed under the terms of the Creative Commons Attribution Non-Commercial License (http://creativecommons.org/licenses/by-nc/4.0) which permits unrestricted non-commercial use, distribution, and reproduction in any medium, provided the original work is properly cited.
} 
versity hospital were included. Subjects with a positive FIT result were offered colonoscopy. All participants were notified of their FIT results via mailings, and only participants with a positive FIT result were invited for a colonoscopy. All information for demographics, colonoscopy, and histopathology was extracted from participants who completed the NCSP between 2009 and 2015 at our center. Subjects were excluded from the analysis if they were aged less than 50 years, were currently hospitalized, refused to participate in this program, or had symptoms or signs indicating the need for a colonoscopy. Subjects were also excluded if they had been diagnosed with inflammatory bowel disease, CRC, or had a history of bowel resection. The Institutional Review Board of Kyung Hee University Hospital at Gangdong reviewed and approved our study protocol (KHNMC IRB number: 2016-11-012) and performed in accordance with the principles of the Declaration of Helsinki. Informed consent was waived for this retrospective study.

\section{Fecal immunochemical test}

FIT samples were collected by visit under NCSP. All participants were invited to our center and made an appointment to revisit the hospital for returning of fecal sample. Participants were instructed to perform sampling for the FIT test on the day of revisit. Most fecal samples were collected on the day of revisit under rapid return system in Korea.

The quantitative FIT was performed using the OC-Sensor DIANA Micro desktop analyzer (Eiken Chemical Co., Tokyo, Japan). ${ }^{8}$ According to the manufacturer instructions, a cutoff value of $20 \mu \mathrm{g} \mathrm{Hb} / \mathrm{g}$ feces (100 ng $\mathrm{Hb} / \mathrm{mL}$ buffer) was used as the criterion of positive FIT. This value was used in the majority of previous studies. ${ }^{9-11}$ The FIT result was expressed in $\mu \mathrm{g}$ of hemoglobin $(\mathrm{Hb})$ per gram of feces rather than nanogram of $\mathrm{Hb}$ per milliliter of buffer in this study. ${ }^{12}$ During the study period, there was no change in the quantitative FIT analyzer, examination methods or cutoff values. Participants were instructed to sample their stool before the contact of stool with water or urine; however, no restrictions in the diet or use of medication before stool sampling were imposed. Participants were instructed to return the sample as soon as possible.

\section{Colonoscopy}

Under NCSP, a confirmatory colonoscopy was provided as a second step for subjects with a positive FIT result. Colonoscopy was performed by gastroenterologists using a standard video colonoscope (CF-H260AI; Olympus Optical, Tokyo, Japan). All participants underwent a 3-day dietary restriction and bowel preparation with split regimen using $4 \mathrm{~L}$ of polyethylene glycol solution (Taejoon Co., Seoul, Korea) or $90 \mathrm{~mL}$ of sodium phosphate (Unimed Co., Seoul, Korea). An established accreditation program was mandatory for endoscopists to practice in the NCSP. A complete colonoscopy was defined as an intubation of the cecum with identification of the ileocecal valve and ap- pendiceal orifice or intubation up to an obstructing neoplasm. A neoplastic lesion located in the proximal colon (including the cecum, ascending colon, transverse colon, and splenic flexure) was defined as proximal colonic neoplasia. ${ }^{13-15}$

\section{Performance measures}

Participation rate of FIT in this study was calculated as the numbers of screenee who submitted stool specimens divided by the numbers of screenee who visited our hospital and received any of the NCSP program. Individual FIT data routinely included the following information: patient demographics, FIT completion rate, positive rate and quantitative value of FIT, colonoscopy completion rate after a positive FIT, and performance of FIT for CRC and advanced neoplasia. For subjects with a positive FIT, individual colonoscopy data also included the following information: patient demographics, colonoscopist's data (staff/fellow status and annual number of performed colonoscopies), waiting time for colonoscopy appointment, quality of bowel preparation, conscious sedation, cecal intubation, withdrawal time, polyp-retrieval rate, polyp detection rate (PDR), adenoma detection rate (ADR), and complications. We considered bowel preparation quality as adequate if the Boston Bowel Preparation Scale $\geq 6$ points (i.e., Boston Bowel Preparation Scale scores of 2 or 3 for all colon segments) as it was adequate for the detection of polyps $>5 \mathrm{~mm}$ in size. ${ }^{16}$ Histopathological data included the number and characteristics of diagnosed polyps/masses, including morphology, location, size, and histology. Based on the most advanced detected lesion, colonoscopic data were classified as normal, any neoplasia, or advanced neoplasia. Advanced neoplasia included all cases of advanced adenomas (an adenoma with high-grade dysplasia, a diameter $\geq 10 \mathrm{~mm}$, or containing $>25 \%$ villous features) or carcinomas. ${ }^{17,18}$ We examined the FIT rate of positive results, detection of CRC, and positive predictive value (PPV) for CRC or advanced neoplasia. PPV of FIT was determined as the number of patients with CRC or advanced neoplasia per the number of patients with positive FIT results. Detection rate of FIT was determined as the number of patients with CRC or advanced neoplasia per the number of patients who received colonoscopy.

\section{Statistical analysis}

Data were expressed as means \pm standard deviation or numbers (percentage). The relation between the FIT quantitative value and advanced neoplasia was evaluated by binary logistic regression with odds ratios (OR) and 95\% confidence intervals (CI). A two-tailed p-value $<0.05$ was considered statistically significant. Statistical analysis was performed using the SPSS version 18.0 for Windows (SPSS Inc., Chicago, IL, USA).

\section{RESULTS}

Table 1 shows the demographic characteristics of the study 
population included in the NCSP. During the study period, 51,439 individuals were invited to participate in the NCSP at our center. Among them, 38,631 individuals (75.1\%) participated in the FIT: their mean age was 61.3 years, and 36.9\% were male. Among 439 patients with positive FIT results, 301 patients (68.6\%) underwent colonoscopy examination (mean age, 63.2 years; 53.5\% male). During the study period, annual FIT completion rate was above $80 \%$ ( $80.5 \%$ to $86.7 \%$ ), and colonoscopy completion rate ranged between $61.4 \%$ and $92.2 \%$.

\section{Performance of fecal immunochemical tests}

The performances of FIT for participants who completed the NCSP are outlined in Table 2. The positive rate of the FIT was

Table 1. Demographic Characteristics of the Study Population Who Underwent the National Cancer Screening Program

\begin{tabular}{lc}
\hline \multicolumn{1}{c}{ Characteristic } & Result \\
\hline Invited persons & \\
No. of invited persons & 51,439 \\
Age, yr & $61.0 \pm 7.7$ \\
Male sex & $17,965(34.9)$ \\
Participants who performed FIT & \\
FIT completion rate & $38,631(75.1)$ \\
Age, yr & $61.3 \pm 7.8$ \\
Male sex & $14,235(36.9)$ \\
Colonoscopy participants & \\
Colonoscopy completion rate & $301(68.6)$ \\
Age, yr & $63.2 \pm 8.0$ \\
Male sex & $161(53.5)$ \\
\hline
\end{tabular}

Data are presented as mean \pm SD or number $(\%)$.

FIT, fecal immunochemical test.

Table 2. Performance of the Fecal Immunochemical Test in Participants Who Underwent the National Cancer Screening Program

\begin{tabular}{lc}
\hline \multicolumn{1}{c}{ Performance of FIT } & Result \\
\hline No. of invited persons & 51,439 \\
Completion rate of FIT & $38,631(75.1)$ \\
Positive rate of FIT & $439(1.1)$ \\
$\quad$ Sex (men vs women) & $220(50.0)$ vs $219(50.0)$ \\
$\quad$ Age (50-64 yr vs $\geq 65$ yr) & $240(54.7)$ vs $199(45.3)$ \\
Quantitative values, $\mu \mathrm{g}$ Hb/g & $68.7 \pm 54.5$ \\
Colonoscopy completion rate after FIT positive & $301(68.6)$ \\
Performance of FIT & \\
Detection rate of CRC & $24(0.1)$ \\
PPV of FIT for CRC, \% & 5.5 \\
Detection rate of advanced neoplasia & $84(0.2)$ \\
PPV of FIT for advanced neoplasia, \% & 19.1
\end{tabular}

Data are presented as number (\%) or mean \pm SD.

FIT, fecal immunochemical test; Hb, hemoglobin; CRC, colorectal cancer; PPV, positive predictive value.
1.1\%, and mean quantitative value of the positive FIT was 68.7 $\mu \mathrm{g} \mathrm{Hb} / \mathrm{g}$. Among 439 patients who had a positive FIT result, 301 patients (68.6\%) underwent colonoscopy examinations. For FIT performance analysis, PPV of CRC and advanced neoplasia were $5.5 \%$ and $19.1 \%$, respectively.

\section{Performance of colonoscopy}

The performance of colonoscopy for patients with positive FIT results in NCSP is outlined in Table 3. Of 301 colonoscopies, $71.1 \%$ colonoscopies were performed by fellow endoscopists, and 29.2\% colonoscopies were performed by endoscopists who had an annual colonoscopy volume $\geq 200$ colonoscopies. Mean waiting time from FIT result mailing to colonoscopy was 39.7

Table 3. Performance of Colonoscopy in Participants with a Positive Fecal Immunochemical Test in the National Cancer Screening Program

\begin{tabular}{|c|c|}
\hline Performance of colonoscopy & Result \\
\hline \multicolumn{2}{|l|}{ Data of colonoscopists } \\
\hline Status (staff) & 87 (28.9) \\
\hline No. of colonoscopies $\geq 200 / y r$ & $88(29.2)$ \\
\hline \multicolumn{2}{|l|}{ Quality indicators of colonoscopy } \\
\hline Colonoscopy completion rate & $301(68.6)$ \\
\hline Waiting time (day) to colonoscopy & $39.7 \pm 46.8$ \\
\hline$\leq 30$ day & $195(64.8)$ \\
\hline$\leq 6 \mathrm{mo}$ & $290(96.3)$ \\
\hline Information and consent & $301(100)$ \\
\hline Adequate bowel preparation & $273(90.7)$ \\
\hline Withdrawal time & $13.3 \pm 7.0$ \\
\hline$\geq 6 \min$ & $282(93.7)$ \\
\hline Cecal intubation rate & $295(98.0)$ \\
\hline Conscious sedation & $186(61.8)$ \\
\hline \multicolumn{2}{|l|}{ Performance of colonoscopy after FIT positive } \\
\hline Polyp-retrieval rate & $157(100)$ \\
\hline Polyp detection rate & $194(64.5)$ \\
\hline Adenoma detection rate, $\%$ & 48.2 \\
\hline Sex (men vs women) & 60.0 vs 33.6 \\
\hline Specialty (staff vs fellow) & 69.6 vs 39.7 \\
\hline Detection rate of CRC, \% & 8.0 \\
\hline Sex (men vs women) & 4.0 vs 4.0 \\
\hline Age (50-64 yr vs $\geq 65$ yr) & 4.7 vs 3.3 \\
\hline Detection rate of advanced neoplasia, \% & 27.9 \\
\hline Sex (men vs women) & 19.3 vs 8.6 \\
\hline Age (50-64 yr vs $\geq 65$ yr) & 14.6 vs 13.3 \\
\hline \multicolumn{2}{|l|}{ Complication rate } \\
\hline Major complications (bleeding, perforation) & 0 \\
\hline Minor complications* & $3(1.0)$ \\
\hline
\end{tabular}

Data are presented as number (\%) or mean \pm SD.

FIT, fecal immunochemical test; CRC, colorectal cancer.

*Minor complications include three cases of transient hypoxia. 
Table 4. Histopathological Characteristics Detected in the National Cancer Screening Program

\begin{tabular}{ll}
\hline Histopathological characteristic & Result \\
\hline FIT analysis ( $\mathrm{n}=38,631)$ & \\
Advanced neoplasia & $24(0.2)$ \\
Colorectal cancer & \\
Colonoscopy analysis ( $\mathrm{n}=301)$ & $84(27.9)$ \\
Advanced neoplasia & $24(8.0)$ \\
Colorectal cancer & \\
Advanced neoplasia analysis $(\mathrm{n}=84)$ & $25(29.8)$ \\
Location (proximal) & $15(3-70)$ \\
Size, mm & $3.1 \pm 3.6$ \\
No. of concurrent adenomas & $75(89.3)$ \\
Shape (polypoid)
\end{tabular}

Data are presented as number (\%), median (range), or mean \pm SD. FIT, fecal immunochemical test.

days, and 96.3\% of subjects underwent colonoscopy within 6 months. Quality indicators of colonoscopy were generally excellent in NCSP and were reflected by 100\% consent rate, 90.7\% adequate preparation, 93.7\% optimal ( $\geq 6$ minutes) withdrawal time, and 98.0\% cecal intubation rate. Additionally, the performance of colonoscopy was generally good and was reflected by $100 \%$ polyp-retrieval rate, $64.5 \%$ rate of PDR, and $48.2 \%$ rate of $\mathrm{ADR}$. ADRs were significantly higher in men than in women (60.0\% vs 33.6\%, $\mathrm{p}=0.000$ ), and in staff endoscopist group than in fellow endoscopist group (69.6\% vs 39.7\%, p=0.000), respectively. During the colonoscopy, no subject experienced major complications, such as bleeding or perforation. However, three patients (1.0\%) experienced transient hypoxia during conscious endoscopy.

\section{Histopathological characteristics}

Histopathological characteristics detected in the NCSP are summarized in Table 4. The analysis of FIT results showed that the detection rate of CRC and advanced neoplasia was 0.1\% and $0.2 \%$, respectively. The colonoscopy analysis showed that the detection rate of CRC and advanced neoplasia was 8.0\% and $27.9 \%$, respectively. The most common advanced neoplasia location detected in the NCSP was distal location (70.2\%) and the most common shape was polypoid (89.3\%).

\section{Performance of CRC screening according to quantitative FIT}

Table 5 shows the performance of NCSP according to quantitative value of FIT. We focused on the data of advanced neoplasia as only advanced neoplasia was regarded as a clinically relevant finding and the most appropriate target for CRC screening. When quantitative value of FIT was divided into three groups, highest tertile group showed significantly higher detection rate of advanced neoplasia than lowest tertile group (OR, 2.6; 95\%
Table 5. Performance of the Screening Program according to the Quantitative Value of Fecal Immunochemical Test

\begin{tabular}{lccc}
\hline \multicolumn{1}{c}{ FIT value, $\mu \mathrm{g} \mathrm{Hb} / \mathrm{g}$} & $\begin{array}{c}\text { Advanced neoplasia } \\
\text { No. }(\%)\end{array}$ & $\begin{array}{c}\text { OR } \\
(95 \% \mathrm{CI})\end{array}$ & $\mathrm{p}$-value \\
\hline Lowest tertile (20-35.8) & $18(21.4)$ & Reference & - \\
Middle tertile (35.9-90.6) & $29(34.5)$ & $1.9(1.0-3.6)$ & 0.07 \\
Highest tertile ( $\geq 90.7)$ & $37(44.0)$ & $2.6(1.4-5.1)$ & $<0.001$ \\
\hline
\end{tabular}

FIT, fecal immunochemical test; OR, odds ratio; CI, confidence interval; $\mathrm{Hb}$, hemoglobin.

CI, 1.4 to $5.1 ; \mathrm{p}<0.001)$.

\section{DISCUSSION}

The U.S. Multi-Society Task Force (USMSTF) on CRC suggested key quality metrics for FIT screening: $\geq 60 \%$ FIT completion rate, $\geq 80 \%$ colonoscopy completion rate for those with a positive FIT, and positive rate of FIT. ${ }^{5}$ In the present study, we proposed detection rate and PPV of CRC or advanced neoplasia as well as completion rate of FIT and colonoscopy as quality metrics in the FIT-based CRC screening program. The USMSTF recommended quantitative FIT rather than qualitative FIT with a low cutoff $(<20 \mu \mathrm{g} \mathrm{Hb} / \mathrm{g}))^{5}$ which was consistent with our program. FIT completion rate of USMSTF was based on $48.2 \%$ to $62.6 \%$ FIT completion rate in previous studies. ${ }^{19-26}$ However, FIT completion rates were variable in each country: for example, $62.6 \%$ in the Netherlands, ${ }^{21} 48.2 \%$ in California (USA), ${ }^{26}$ $40.7 \%$ in Texas (USA), ${ }^{27} 34.2 \%$ in Spain, ${ }^{28}$ and 32.3\% in Italy. ${ }^{29}$ Furthermore, FIT completion rates were also variable through different strategies of CRC screening. ${ }^{30}$ Considering low FIT completion rate reported during 2009 to 2013 in Korea, ${ }^{31}$ our high FIT completion rate may be reflected by recent quality improvement in NCSP. Therefore, the optimal level of FIT completion rate should be settled in each country.

The USMSTF guideline suggested a "positive rate of FIT" as a potential quality metrics. ${ }^{5}$ Positive rate of FIT ranged between $4.3 \%$ and $10.1 \%$ in previous studies. ${ }^{19-26}$ The positive rate of FIT was $10.1 \%$ in a biennial FIT with postal invitations in Ireland ${ }^{22}$ and $4.3 \%$ in a prospective cohort study in Italy. ${ }^{19}$ In NCSP of Korea, the reported positive rates of FIT ranged between $7.1 \%$ and 7.9\%, which were mixed results of qualitative and quantitative tests. ${ }^{32}$ However, positive rate of quantitative FIT was only $2.0 \%$ in a prospective NCSP setting in Korea. ${ }^{33}$ In our previous study, quantitative FIT positive rate was only 1.3\%, which was similar to the results $(1.1 \%)$ of the current study. Considering the higher accuracy of quantitative FIT and different positive rates between the quantitative and qualitative tests, ${ }^{34}$ the optimal target of quantitative FIT positive rate should be suggested for FIT-based screening program.

USMSTF also suggested quality metrics for FIT-based colonoscopy: $\geq 80 \%$ colonoscopy completion rate after positive FIT 
results and $>35 \%$ to $45 \%$ ADR ( $>45 \%$ in men and $>35 \%$ in women) on colonoscopy after FIT positive. ${ }^{5}$ In our study, we also suggested additional quality metrics for colonoscopy after a positive FIT: colonoscopist's specialty and waiting time between the notification of a positive FIT result and the colonoscopy (Table 3). European Society of Gastrointestinal Endoscopy (ESGE) suggested a minimum annual number of screening colonoscopies for the specialty of screening colonoscopists. ${ }^{4}$ The English Bowel Cancer Screening Program requires a minimum annual number of 150 screening colonoscopies, ${ }^{35}$ which was based on the findings a threefold increased risk of complications if colonoscopists performed fewer than 300 colonoscopies per year. ${ }^{36}$ Spanish Society of Gastrointestinal Endoscopy (SSGE) working group also recommends a minimum experience in colonoscopy (at least 200 supervised colonoscopies) and continuous dedication of colonoscopists (at least 200 colonoscopies per year) for the specialty of screening colonoscopists. ${ }^{7}$ Therefore, a minimum annual number of screening colonoscopies for the specialty of screening colonoscopists should be suggested in the further studies. The U.S. and European guidelines did not suggest the optimal waiting time between a FIT positive result and colonoscopy. ${ }^{4,5}$ However, SSGE working group recommends an optimal waiting time for a colonoscopy $<6$ weeks. ${ }^{7}$ In a study on U.S. health care system, waiting time to colonoscopy was less than 6 months in most patients. ${ }^{37}$ Using a microsimulation model of an average-risk U.S. screening cohort, waiting time up to 12 months can produce a proportional decrease of up to $10 \%$ in overall screening benefit. ${ }^{38}$ In 231 patients with positive FIT, longer waiting time to colonoscopy was associated with higher OR of neoplasia (OR=1.10 for additional 30-day waiting time). ${ }^{39}$ In our study, average waiting time to colonoscopy was only 39.7 days, which was shorter than those reported in previous studies and recommendation. ${ }^{7,38,39}$ Considering the importance of timely follow-up colonoscopy after a positive FIT, an optimal waiting time for colonoscopy, such as 6 months, should be included as a quality metrics of FIT-based screening. From the perspective of quality indicators of screening colonoscopy, some quality metrics such as $>95 \%$ adequate preparation, ${ }^{40} \geq 95 \%$ cecal intubation, ${ }^{40} \geq 6$ minutes withdrawal time, ${ }^{40}$ perforation rate $<1: 1,000$ and post-polypectomy bleeding $<1: 100-200^{4,7,40}$ may be equally adopted for FIT-based colonoscopy as well as primary screening colonoscopy.

The most important measure of a FIT-based screening is neoplasia detection rate. However, this parameter was not suggested as quality metrics by USMSTF due to low likelihood of those findings and the progressive reduction of those findings with subsequent round of FIT testing. ${ }^{5}$ In this study, we suggested PDR, ADR, and detection rate of CRC or advanced neoplasia as quality metrics for a FIT-based screening as they are target outcomes of CRC screening program. In previous studies, PPV of CRC ranged between 2.9\% and 7.8\% and PPV of advanced neoplasia ranged between 32.9\% and 54.0\%. ${ }^{19-26}$ Our study showed similar PPV of CRC (5.5\%), but lower PPV (19.1\%) of advanced neoplasia, compared with those of previous studies. ${ }^{19-26}$ In terms of detection rate of neoplasia, a prospective, multicenter study showed a detection rate of CRC and advanced neoplasia of 8.1\% and $40.1 \%$, respectively. ${ }^{30}$ Our study showed similar detection rate of CRC (8.0\%), but lower (27.9\%) detection rate of advanced neoplasia, compared with this study. ${ }^{30}$ The USMSTF suggested $>35 \%-45 \%$ ADR (>45\% in men and >35\% in women) and the SSGE working group recommended $>40 \%$ ADR on colonoscopy after positive FIT results. ${ }^{5,7}$ The ADR by colonoscopy after a positive FIT result was 35\% in an Irish cohort, ${ }^{22} 51.5 \%$ in a U.S. study, ${ }^{26}$ and $48.2 \%$ in our study. Therefore, $>40 \%$ ADR on colonoscopy after a positive FIT result seems to be optimal. Our study showed that highest tertile of FIT level can predict higher detection rate of advanced neoplasia than lowest tertile level (OR, 2.6; 95\% CI, 1.4 to 5.1; $\mathrm{p}<0.001$ ). Our findings can be confirmed by the fact that patients with CRC or advanced neoplasia had significantly higher FIT levels $(p<0.001)$ than patients without these lesions. ${ }^{41}$ In a Taiwan CRC screening program, ${ }^{42}$ a significant log-linear relationship was found between the level of FIT and PPV for colorectal tumors. Considering only 68.6\% colonoscopy completion rate in the present study, colonoscopy should be strongly recommended in patients with a high FIT level in order to detect a potential advanced neoplasia. ${ }^{43}$

Our study has some advantages. Our current study is the first study to evaluate the performance and quality metrics of FITbased CRC screening program in Korea. Our study may support the fact that some quality metrics used in the United States and Europe $e^{4,6,7}$ may be directly introduced to other countries, including Korea. Furthermore, our study was based on accurate, reliable, manually collected data involving high-quality colonoscopy. Nonetheless, this study also had some limitations. First, an inherent limitation of our study is the possibility of a selection bias from observational, retrospective design from a single university hospital. Higher participation of fellow colonoscopists in our study may also influence the performance of colonoscopy. Second, we could not calculate the sensitivity and specificity of FIT-based screening and the performance of FIT in the participants with negative FIT results, as only participants with a positive FIT result were invited back for a colonoscopy. Third, our low positive rate of FIT, PPV of advanced neoplasia and low colonoscopy completion rate may be explained because participants who recently received colonoscopy were not excluded from the NCSP of Korea. In addition, low colonoscopy completion rate may be caused by some colonoscopy data was missed from NCSP database as some patients with a positive FIT result underwent colonoscopy at their own expense as accessibility of colonoscopy is excellent with low cost in Korea. Fourth, the proportion of unacceptable samples for FIT test by the laboratory, which was suggested as $<5 \%$ by USMSTF, ${ }^{5}$ was not evaluated in this study. However, it may be extremely rare in NCSP, as all subjects returned their fecal samples by visit on the day 
of revisit. For example, the number of unacceptable samples for FIT test was 0 in 1,191 fecal samples in December 2016. Finally, we could not examine the relationship between quality metrics of FIT-based screening and the clinical outcomes, such as mortality of CRC or risk of interval cancer.

In conclusion, quality metrics used in the United States and Europe may be directly introduced to other countries, including Korea. However, the optimal quality metrics should be settled in each country and future studies on this issue should be warranted.

\section{CONFLICTS OF INTEREST}

No potential conflict of interest relevant to this article was reported.

\section{REFERENCES}

1. Ferlay J, Soerjomataram I, Dikshit R, et al. Cancer incidence and mortality worldwide: sources, methods and major patterns in GLOBOCAN 2012. Int J Cancer 2015;136:E359-E386.

2. Shin A, Choi KS, Jun JK, et al. Validity of fecal occult blood test in the national cancer screening program, Korea. PLoS One 2013;8:e79292.

3. Yoo KY. Cancer control activities in the Republic of Korea. Jpn J Clin Oncol 2008;38:327-333.

4. Rembacken B, Hassan C, Riemann JF, et al. Quality in screening colonoscopy: position statement of the European Society of Gastrointestinal Endoscopy (ESGE). Endoscopy 2012;44:957-968.

5. Robertson DJ, Lee JK, Boland CR, et al. Recommendations on fecal immunochemical testing to screen for colorectal neoplasia: a consensus statement by the US Multi-Society Task Force on colorectal cancer. Gastrointest Endosc 2017;85:2-21.e3.

6. Rizk MK, Sawhney MS, Cohen J, et al. Quality indicators common to all GI endoscopic procedures. Gastrointest Endosc 2015;81:316.

7. Jover R, Herráiz M, Alarcón 0, et al. Clinical practice guidelines: quality of colonoscopy in colorectal cancer screening. Endoscopy 2012;44:444-451.

8. Jeon CH, Lee AJ, Kim KD. Annual report on external quality assessment scheme for urinalysis and faecal occult blood testing in Korea. J Lab Med Qual Assur 2015;37:179-189.

9. Omata F, Shintani A, Isozaki M, Masuda K, Fujita Y, Fukui T. Diagnostic performance of quantitative fecal immunochemical test and multivariate prediction model for colorectal neoplasms in asymptomatic individuals. Eur J Gastroenterol Hepatol 2011;23:1036-1041.

10. Fraser CG, Allison JE, Halloran SP, Young GP; Expert Working Group on Fecal Immunochemical Tests for Hemoglobin, Colorectal Cancer Screening Committee, World Endoscopy Organization. A proposal to standardize reporting units for fecal immunochemical tests for hemoglobin. J Natl Cancer Inst 2012;104:810-814.
11. Sohn DK, Jeong SY, Choi HS, et al. Single immunochemical fecal occult blood test for detection of colorectal neoplasia. Cancer Res Treat 2005;37:20-23.

12. Fenocchi E, Martínez L, Tolve J, et al. Screening for colorectal cancer in Uruguay with an immunochemical faecal occult blood test. Eur J Cancer Prev 2006;15:384-390.

13. Lin OS, Schembre DB, McCormick SE, et al. Risk of proximal colorectal neoplasia among asymptomatic patients with distal hyperplastic polyps. Am J Med 2005;118:1113-1119.

14. Ruco A, Stock D, Hilsden RJ, et al. Evaluation of a risk index for advanced proximal neoplasia of the colon. Gastrointest Endosc 2015;81:1427-1432.

15. Schreiner MA, Weiss DG, Lieberman DA. Proximal and large hyperplastic and nondysplastic serrated polyps detected by colonoscopy are associated with neoplasia. Gastroenterology 2010;139:1497-1502.

16. Clark BT, Protiva P, Nagar A, et al. Quantification of adequate bowel preparation for screening or surveillance colonoscopy in men. Gastroenterology 2016;150:396-405.

17. Grazzini G, Castiglione G, Ciabattoni C, et al. Colorectal cancer screening programme by faecal occult blood test in Tuscany: first round results. Eur J Cancer Prev 2004;13:19-26.

18. Rozen P, Levi Z, Hazazi R, et al. Quantitative colonoscopic evaluation of relative efficiencies of an immunochemical faecal occult blood test and a sensitive guaiac test for detecting significant colorectal neoplasms. Aliment Pharmacol Ther 2009;29:450-457.

19. Crotta S, Segnan N, Paganin S, Dagnes B, Rosset R, Senore C. High rate of advanced adenoma detection in 4 rounds of colorectal cancer screening with the fecal immunochemical test. Clin Gastroenterol Hepatol 2012;10:633-638.

20. Denters MJ, Deutekom M, Bossuyt PM, Stroobants AK, Fockens P, Dekker E. Lower risk of advanced neoplasia among patients with a previous negative result from a fecal test for colorectal cancer. Gastroenterology 2012;142:497-504.

21. Kapidzic A, Grobbee EJ, Hol L, et al. Attendance and yield over three rounds of population-based fecal immunochemical test screening. Am J Gastroenterol 2014;109:1257-1264.

22. McNamara D, Leen R, Seng-Lee C, et al. Sustained participation, colonoscopy uptake and adenoma detection rates over two rounds of the Tallaght-Trinity College colorectal cancer screening programme with the faecal immunological test. Eur J Gastroenterol Hepatol 2014;26:1415-1421.

23. Parente F, Boemo C, Ardizzoia A, et al. Outcomes and cost evaluation of the first two rounds of a colorectal cancer screening program based on immunochemical fecal occult blood test in northern Italy. Endoscopy 2013;45:27-34.

24. Stegeman I, van Doorn SC, Mundt MW, et al. Participation, yield, and interval carcinomas in three rounds of biennial FIT-based colorectal cancer screening. Cancer Epidemiol 2015;39:388-393.

25. van Roon AH, Goede SL, van Ballegooijen M, et al. Random comparison of repeated faecal immunochemical testing at different intervals for population-based colorectal cancer screening. Gut 
2013;62:409-415.

26. Jensen CD, Corley DA, Quinn VP, et al. Fecal immunochemical test program performance over 4 rounds of annual screening: a retrospective cohort study. Ann Intern Med 2016;164:456-463.

27. Gupta S, Halm EA, Rockey DC, et al. Comparative effectiveness of fecal immunochemical test outreach, colonoscopy outreach, and usual care for boosting colorectal cancer screening among the underserved: a randomized clinical trial. JAMA Intern Med 2013;173:1725-1732.

28. Quintero E, Castells A, Bujanda L, et al. Colonoscopy versus fecal immunochemical testing in colorectal-cancer screening. N Engl J Med 2012;366:697-706.

29. Segnan N, Senore C, Andreoni B, et al. Comparing attendance and detection rate of colonoscopy with sigmoidoscopy and FIT for colorectal cancer screening. Gastroenterology 2007;132:23042312.

30. Segnan N, Senore C, Andreoni B, et al. Randomized trial of different screening strategies for colorectal cancer: patient response and detection rates. J Natl Cancer Inst 2005;97:347-357.

31. Suh M, Choi KS, Park B, et al. Trends in cancer screening rates among Korean men and women: results of the Korean National Cancer Screening Survey, 2004-2013. Cancer Res Treat 2016;48:110.

32. Park MJ, Choi KS, Jun JK, Lee HY. Trends in the National Cancer Screening Program for colorectal cancer in the Republic of Korea, 2004-2009. Asian Pac J Cancer Prev 2011;12:3489-3493.

33. Shin HY, Suh M, Baik HW, et al. Performance of the fecal immunochemical test for colorectal cancer screening using different stool-collection devices: preliminary results from a randomized controlled trial. Gut Liver 2016;10:925-931.

34. Park MJ, Choi KS, Lee YK, Jun JK, Lee HY. A comparison of quali- tative and quantitative fecal immunochemical tests in the Korean national colorectal cancer screening program. Scand J Gastroenterol 2012;47:461-466.

35. NHS Bowel Cancer Screening Programme Quality Assurance Endoscopy Group. Quality assurance guidelines for colonoscopy. Sheffield: NHS Cancer Screening Programmes, 2011.

36. Rabeneck L, Paszat LF, Hilsden RJ, et al. Bleeding and perforation after outpatient colonoscopy and their risk factors in usual clinical practice. Gastroenterology 2008;135:1899-1906.

37. Chubak J, Garcia MP, Burnett-Hartman AN, et al. Time to colonoscopy after positive fecal blood test in four U.S. health care systems. Cancer Epidemiol Biomarkers Prev 2016;25:344-350.

38. Meester RG, Zauber AG, Doubeni CA, et al. Consequences of increasing time to colonoscopy examination after positive result from fecal colorectal cancer screening test. Clin Gastroenterol Hepatol 2016;14:1445-1451.e8.

39. Gellad ZF, Almirall D, Provenzale D, Fisher DA. Time from positive screening fecal occult blood test to colonoscopy and risk of neoplasia. Dig Dis Sci 2009;54:2497-2502.

40. Rex DK, Schoenfeld PS, Cohen J, et al. Quality indicators for colonoscopy. Am J Gastroenterol 2015;110:72-90.

41. Hernandez V, Cubiella J, Gonzalez-Mao MC, et al. Fecal immunochemical test accuracy in average-risk colorectal cancer screening. World J Gastroenterol 2014;20:1038-1047.

42. Chen $\mathrm{CH}$, Wen CP, Tsai MK. Fecal immunochemical test for colorectal cancer from a prospective cohort with 513,283 individuals: providing detailed number needed to scope (NNS) before colonoscopy. Medicine (Baltimore) 2016;95:e4414.

43. Liao CS, Lin YM, Chang HC, et al. Application of quantitative estimates of fecal hemoglobin concentration for risk prediction of colorectal neoplasia. World J Gastroenterol 2013;19:8366-8372. 